Research Paper

\title{
Impact of Detection Method and Accompanying Ductal Carcinoma in Situ on Prognosis of Tla,bNO Breast Cancer
}

\author{
Shih-Che Shen ${ }^{1}$, Shir-Hwa Ueng ${ }^{2}$, Chan-Keng Yang ${ }^{3}$, Chi-Chang Y $\mathbf{u}^{1}$, Yung-Feng Lo', Hsien-Kun Chang ${ }^{3}$, \\ Yung-Chang Lin $^{3}$, Shin-Cheh Chen ${ }^{1}$ \\ 1. Department of Surgery, Chang Gung Memorial Hospital, Chang Gung University Medical College, No. 5, Fu Hsing St., Guishan Dist., 33305, Taoyuan, \\ Taiwan \\ 2. Department of Pathology, Chang Gung Memorial Hospital, Chang Gung University Medical College, No. 5, Fu Hsing St., Guishan Dist., 33305, Taoyuan, \\ Taiwan \\ 3. Department of Oncology, Chang Gung Memorial Hospital, Chang Gung University Medical College, No. 5, Fu Hsing St., Guishan Dist., 33305, Taoyuan, \\ Taiwan
}

$\square$ Corresponding author: Dr. Shin-Cheh Chen, Department of Surgery, Chang Gung Memorial Hospital, Chang Gung University Medical College, No. 5, Fu Hsing St., Guishan Dist., 33305 Taoyuan City, Taiwan. Tel: + 886-2-27135211, ext 3141 | Fax: + 886-3-3285818; E-mail: chensc@adm.cgmh.org.tw

(c) Ivyspring International Publisher. This is an open access article distributed under the terms of the Creative Commons Attribution (CC BY-NC) license (https://creativecommons.org/licenses/by-nc/4.0/). See http://ivyspring.com/terms for full terms and conditions.

Received: 2017.01.22; Accepted: 2017.04.01; Published: 2017.07.21

\begin{abstract}
Background: $\mathrm{Tla}$, bNO breast cancer is not easily detected. Before mammography became widespread, most cases were discovered only after the development of symptoms. The presence of ductal carcinoma in situ (DCIS) affects the detectability of associated invasive cancer; however, the prognostic value of concomitant DCIS is controversial. This study compared the characteristics of screening-detected and symptom-detected Tla,bNO breast cancer, and investigated the impact of accompanying DCIS on detection and prognosis.

Patients and Methods: Data were collected from a single hospital between 2000 and 2009. Of 5,690 primary breast cancers patients, 438 met the criteria for Tla,bNOMO. Logistic regression models were used to identify prognostic indicators and their association with the detection method. Survival analyses were performed to estimate distant relapse-free survival (DRFS) and breast cancer-specific survival (BCSS).

Results: Tumors in 79 and 359 patients were detected by screening and development of symptoms, respectively. Symptomatic cancer patients were younger, more likely to receive a mastectomy, and had larger accompanying DCIS lesions; their 10-year DRFS rates were worse than those of patients with screening-detected tumors $(91.1 \%$ vs. $100 \%$ respectively, $p=0.049)$. Patients with large accompanying DCIS $(\geq 2 \mathrm{~cm})$ had markedly worse 10 -year DRFS $(77.1 \%$ vs. $97.4 \%, p<0.001)$ and BCSS $(94.3 \%$ vs. $98.9 \%, p<0.001)$.

Conclusion: $\mathrm{Tl}$ a,bNO breast cancers detected owing to symptoms are more likely to have larger accompanying DCIS. Tla,bN0 patients with large accompanying DCIS have worse DRFS and BCSS. It is important to consider associated DCIS size when evaluating prognosis in Tla,bNo breast cancer patients.
\end{abstract}

Key words: T1a,bN0 breast cancer; prognosis; accompanying DCIS; cancer screening; asymptomatic disease

\section{Introduction}

The characterization and survival of patients with stage $\mathrm{T} 1 \mathrm{a}, \mathrm{bN0}$ breast cancer have garnered increased attention in recent years; several reports have been published on the prognoses and treatment outcomes of this disease. However, the reported prognoses for $\mathrm{T} 1 \mathrm{a}, \mathrm{bN} 0$ breast cancer vary widely, 
partly because studies employ different outcome measurements. In a review of the literature, Hanrahan et al. found that the 10-year distant relapse-free survival rate (DRFS) was above $98 \%$ in some studies, but only $80 \%$ in others [1]. Additionally, most studies evaluated in Hanrahan et al.'s review were performed prior to 1990, before breast cancer screening programs began in many countries. Survival estimates in these studies were likely to be biased, as most T1a,bN0 breast cancer cases today are detected through screening.

With increased use of screening, the diagnosis rates of stage I breast cancer have risen significantly, and now comprise approximately $48 \%$ of all breast cancer cases [2]. In a recently published study on outcomes of T1a,bN0 breast cancer, approximately $75 \%$ of the cases were detected via screening [3]. Favorable outcomes for screening-detected breast cancer have been reported in the literature [4-6]; however, few studies have focused on T1a,bN0 breast cancer. In a study by Lee et al., the 5-year DRFS was only $87 \%$ in palpable $\mathrm{T} 1 \mathrm{a}, \mathrm{bN} 0$ cases, but $100 \%$ in screening-detected lesions $(p=0.02)$ [7].

The detection of sub-centimeter breast tumors is challenging; before the prevalence of mammography screening, most cases were discovered because of an accompanying symptom. It has been suggested that the presence of ductal carcinoma in situ (DCIS) affects the detectability of associated invasive cancer [8]. In a study by Chagpar et al., more than one-quarter of patients with invasive carcinoma had concomitant DCIS [9]. The effect of accompanying DCIS on detectability is even more pronounced when the invasive tumor is small. In studies of T1a,bN0 breast cancer, large DCIS lesions have usually been found adjacent to invasive tumors, especially those that are HER2-positive [10, 11]. In another study of triple-negative $\mathrm{T} 1 \mathrm{a}, \mathrm{bN} 0$ breast cancer, $83 \%$ of the invasive cancers were associated with DCIS [12].

The prognostic impact of DCIS lesions that accompany invasive tumors is controversial. In one study, accompanying DCIS was found to predict improved local recurrence-free survival in breast cancer patients [13]; however, other studies have shown that, while the presence of concomitant DCIS is associated with favorable features, the prognosis is not altered $[8,9]$.

The present study investigated the differences in characteristics between screening-detected and symptom-detected patients, as well as the impact of accompanying DCIS on detection and prognosis in $\mathrm{T} 1 \mathrm{a}, \mathrm{bN} 0$ breast cancer.

\section{Patients and Methods}

There were 5,690 women with invasive breast cancer treated between January 1, 2000 and December 31, 2009 identified in our databank. Patients were selected for our study based on 3 criteria: 1 ) invasive tumor size $\leq 0.5 \mathrm{~cm}$ (T1a) or $>0.5 \mathrm{~cm}$ but $\leq 1.0 \mathrm{~cm}$ (T1b); 2) the patient completed axillary staging and was negative for lymph node metastasis; and 3) the statuses of estrogen receptor (ER), progesterone receptor (PR), and human epidermal growth factor receptor 2 (HER2) were known. Patients who received neoadjuvant therapy or had previous histories of breast malignancy or distant metastasis were excluded. Those who received adjuvant trastuzumab were also excluded because this agent was not recommended for $\mathrm{T} 1 \mathrm{a}, \mathrm{bN} 0$ breast cancer according to our treatment guidelines that were in effect during the study period, although it appears to have an impact on survival according to more recent evidence. Ultimately, 438 patients with T1a,bN0 breast cancer were included.

The following factors were retrieved from our database for analysis: age, invasive tumor size, size of accompanying DCIS, surgical method, Nottingham Bloom Richardson (NBR) grade, immunohistochemistry (IHC) staining of ER, PR, and HER2, and type of adjuvant therapy. The pathologic size of DCIS was divided into large $(\geq 2 \mathrm{~cm})$ or small $(<2 \mathrm{~cm})$ based on the studies by Nair and Lagios [14, 15]. Information on detection method was obtained by reviewing medical records, and cases were divided into 1) screening-detected, where the lesion was detected by either ultrasonography or mammography; and 2) symptomatic, where the tumor was discovered after experiencing a palpable mass, nipple discharge, pain, nipple morphology change, or other signs.

IHC staining of ER, PR, and HER2 was performed by a College of American Pathologists-certified unit at our pathology department. ER and PR were considered positive if $\geq 1 \%$ of tumor cells were stained; HER 2 was defined as positive if more than $30 \%$ of cancer cells showed strong complete membrane staining by IHC, or if fluorescent in situ hybridization yielded a HER2/CEP17 ratio $\geq 2.2$. Luminal A subtype was defined as ER/PR positive, HER2 negative, and NBR grade 1; Luminal $\mathrm{B}$ was defined as ER- and/or PR-positive without fulfilling other Luminal A criteria. The HER2 subtype was negative for ER and PR and positive for the HER2 receptor. The triple negative subtype was negative for ER, PR, and HER2. Adjuvant treatment was recommended according to our treatment guidelines, which have since undergone periodic revisions. Data on adjuvant therapy (including radiation, chemotherapy, and hormonal therapy) were collected. 
Table 1. Characteristics of patients with Tla,bNO breast cancer

\begin{tabular}{|c|c|c|c|c|c|}
\hline \multicolumn{2}{|l|}{ Variable } & \multirow{2}{*}{$\begin{array}{l}\text { T1a,bN0 } \\
\mathrm{n}=438 \\
(\%)\end{array}$} & \multirow{2}{*}{$\begin{array}{l}\text { T1aN0 } \\
\mathrm{n}=164 \\
(\%)\end{array}$} & \multirow{2}{*}{$\begin{array}{l}\text { T1bN0 } \\
\text { n=274 } \\
(\%)\end{array}$} & $\begin{array}{l}p \\
\text { value }\end{array}$ \\
\hline \multirow{2}{*}{ Age (years) } & $\leq 40$ & & & & 0886 \\
\hline & $>40$ & 365 (83.3) & $\begin{array}{l}136 \\
(82.9)\end{array}$ & $\begin{array}{l}229 \\
(83.6)\end{array}$ & \\
\hline \multirow[t]{2}{*}{$\begin{array}{l}\text { Tumor size }(\mathrm{cm}) \text {, median } \\
\text { (IQR) }\end{array}$} & Invasive size & $\begin{array}{l}0.70 \\
(0.51)\end{array}$ & $\begin{array}{l}0.25 \\
(0.30)\end{array}$ & $\begin{array}{l}0.80 \\
(0.30)\end{array}$ & $<0.0001$ \\
\hline & DCIS size & $\begin{array}{l}1.00 \\
(2.50)\end{array}$ & $\begin{array}{l}2.40 \\
(2.64)\end{array}$ & $\begin{array}{l}0.30 \\
(1.40)\end{array}$ & $<0.0001$ \\
\hline \multirow[t]{2}{*}{ DCIS size $(\mathrm{cm})$} & $<2$ & $295(68.3)$ & $70(44.0)$ & $\begin{array}{l}225 \\
(82.4)\end{array}$ & $<0.0001$ \\
\hline & $\geq 2$ & $137(31.7)$ & $89(56.0)$ & 48 (17.6) & \\
\hline \multirow[t]{2}{*}{ Surgery } & Mastectomy & $224(51.1)$ & $96(58.5)$ & $\begin{array}{l}128 \\
(46.7)\end{array}$ & 0.017 \\
\hline & BCS & $214(48.9)$ & $68(41.5)$ & $\begin{array}{l}146 \\
(53.3)\end{array}$ & \\
\hline \multirow[t]{2}{*}{ Detection method } & Screening & $79(18.0)$ & $35(21.3)$ & $44(16.1)$ & 0.164 \\
\hline & Symptomatic & $359(82.0)$ & $\begin{array}{l}129 \\
(78.7)\end{array}$ & $\begin{array}{l}230 \\
(83.9)\end{array}$ & \\
\hline \multirow[t]{3}{*}{ Symptom } & Palpable mass & $329(91.6)$ & $\begin{array}{l}115 \\
(89.1)\end{array}$ & $\begin{array}{l}214 \\
(93.0)\end{array}$ & 0.003 \\
\hline & $\begin{array}{l}\text { Nipple } \\
\text { discharge }\end{array}$ & $22(6.1)$ & $14(10.9)$ & $8(3.5)$ & \\
\hline & Others & $8(2.2)$ & 0 & $8(3.5)$ & \\
\hline \multirow[t]{2}{*}{ NBR grade } & Gr. 1 & $201(47.3)$ & $64(40.5)$ & $\begin{array}{l}137 \\
(51.3)\end{array}$ & 0.031 \\
\hline & Gr. 2,3 & $224(52.7)$ & $94(59.5)$ & $\begin{array}{l}130 \\
(48.7)\end{array}$ & \\
\hline \multirow[t]{2}{*}{ ER } & Negative & $133(30.4)$ & $68(41.5)$ & $65(23.7)$ & $<0.0001$ \\
\hline & Positive & $305(69.6)$ & $96(58.5)$ & $\begin{array}{l}209 \\
(76.3)\end{array}$ & \\
\hline \multirow[t]{2}{*}{ PR } & Negative & $159(36.3)$ & $74(45.1)$ & $85(31.0)$ & 0.003 \\
\hline & Positive & $279(63.7)$ & $90(54.9)$ & $\begin{array}{l}189 \\
(69.0)\end{array}$ & \\
\hline \multirow[t]{2}{*}{ HER2 } & Negative & $316(72.1)$ & $99(60.4)$ & $\begin{array}{l}217 \\
(79.2)\end{array}$ & $<0.0001$ \\
\hline & Positive & $122(27.9)$ & $65(39.6)$ & $57(20.8)$ & \\
\hline \multirow[t]{4}{*}{ Subtypes } & Luminal A & $127(29.5)$ & $33(20.1)$ & $94(34.3)$ & $<0.001$ \\
\hline & Luminal B & $188(43.7)$ & $69(42.1)$ & $\begin{array}{l}119 \\
(44.2)\end{array}$ & \\
\hline & Her2 & $72(16.7)$ & $42(25.6)$ & $30(10.9)$ & \\
\hline & Triple negative & $43(10.0)$ & $17(10.4)$ & $26(9.5)$ & \\
\hline \multirow[t]{2}{*}{ Chemotherapy } & No & $288(65.8)$ & $\begin{array}{l}128 \\
(78.0)\end{array}$ & $\begin{array}{l}160 \\
(58.4)\end{array}$ & $<0.0001$ \\
\hline & Yes & $150(34.2)$ & $36(22.0)$ & $\begin{array}{l}114 \\
(41.6)\end{array}$ & \\
\hline \multirow[t]{2}{*}{ Hormone therapy } & No & $137(31.3)$ & $70(42.7)$ & $67(24.5)$ & $<0.0001$ \\
\hline & Yes & $301(68.7)$ & $94(57.3)$ & $\begin{array}{l}207 \\
(75.5)\end{array}$ & \\
\hline \multirow[t]{2}{*}{ Radiotherapy } & No & $244(55.7)$ & $\begin{array}{l}105 \\
(64.0)\end{array}$ & $\begin{array}{l}139 \\
(50.7)\end{array}$ & 0.007 \\
\hline & Yes & $194(44.3)$ & $59(36.0)$ & $\begin{array}{l}135 \\
(49.3)\end{array}$ & \\
\hline
\end{tabular}

$\mathrm{IQR}=$ Interquartile range

$\mathrm{BCS}=$ Breast conserving surgery

NBR $=$ Nottingham Bloom Richardson

DCIS $=$ Ductal carcinoma in situ

$\mathrm{ER}=$ Estrogen receptor

$\mathrm{PR}=$ Progesterone receptor

HER2 $=$ Human epidermal growth factor receptor 2

We used distant relapse-free survival (DRFS) as the primary endpoint in this study because it corresponded to breast cancer-specific survival (BCSS) and is the foremost indicator when considering adjuvant therapy; the secondary endpoint was BCSS. This study was reviewed and approved by the Ethical Committee of our institution (104-4699B); the review board waived the requirement for written informed consent for all patients.

\section{Statistical Analysis}

The chi-square test was used to determine differences in categorical predictor variables between patients with T1aN0 and T1bN0 breast cancer and between screening-detected and symptomatic T1a,bN0 breast cancer; a Mann-Whitney U test was utilized for continuous predictor variables. Multivariate statistics were used to determine the independent effects of predictor variables on both DRFS and BCSS. DRFS was defined as the time from surgery to distant relapse; BCSS was defined as the time from surgery to death due to breast cancer. We first performed stepwise logistic regression analysis to estimate the associations between prognostic variables and detection methods (symptomatic vs. screening). Survival analysis was performed using the Kaplan-Meier method; survival curves were compared by using a log-rank test where $p<0.05$ was considered statistically significant. All statistical tests were 2-sided. All analyses were performed with the SPSS software (version 16; SPSS, Inc., Chicago, IL).

\section{Results}

Among the 438 cases of T1a,bN0 breast cancer in the study, 164 were T1a and 274 were T1b tumors. Significant differences were noted between T1aN0 and T1bN0 cases (Table 1). Patients with T1aN0 were more likely to have aggressive features such as higher grade $(p=0.031)$, a higher prevalence of ER- or PR-negative status $(p<0.0001$ and $p=0.003$, respectively), and a larger proportion of HER2-positive patients $(p<0.0001)$. Additionally, the sizes of accompanying DCIS lesions tended to be larger than in patients with T1bN0 tumors $(2.4 \mathrm{~cm} v s$. $0.3 \mathrm{~cm}$, respectively; $p<0.0001$ ); T1aN0 tumors were also more frequently accompanied by large $(\geq 2 \mathrm{~cm})$ DCIS lesions $(p<0.0001)$. A higher proportion of T1aN0 patients had received a mastectomy; conversely, adjuvant chemotherapy, hormonal therapy, and radiotherapy were administered more often to T1bN0 patients.

To understand the differences in characteristics between screening-detected (18\%) and symptomatic $(82 \%)$ T1a,bN0 breast cancer patients, pathologic factors were subjected to univariate analysis. Although the invasive tumor size did not differ between screening-detected and symptomatic cases, the latter were more likely to have larger accompanying DCIS lesions $(1.21 \mathrm{~cm}$ vs. $2.37 \mathrm{~cm}$, respectively; $p<0.0001)$, have large DCIS lesions $(16.7 \%$ vs. $35 \%$, respectively; $p=0.002)$, and affect younger $(\leq 40$ years $)$ patients $(6.3 \%$ vs. $18.9 \%$, respectively; $p=0.006$ ) (Table 2). On multivariate analysis, symptomatic $\mathrm{T} 1 \mathrm{a}, \mathrm{bN} 0$ patients were more likely to have large DCIS lesions (odds ratio [OR]: 
$2.00, p=0.043$ ), be of younger age (OR: $3.61, p=0.009$ ), and to have undergone a mastectomy (OR: 2.16, $p=0.006)$ when compared to their screening-detected counterparts (Table 3 ).

Table 2. Differences in characteristics of patients with Tla,bN0 breast cancer by detection method

\begin{tabular}{|c|c|c|c|}
\hline \multirow[t]{2}{*}{ Variable } & \multicolumn{2}{|c|}{ Detection Method } & \multirow{2}{*}{$\begin{array}{l}p \\
p \\
\text { value }\end{array}$} \\
\hline & $\begin{array}{l}\text { Screening } \\
\mathrm{n}=79(\%)\end{array}$ & $\begin{array}{l}\text { Symptomatic } \\
n=359(\%)\end{array}$ & \\
\hline Age (years) & & & 0.006 \\
\hline$\leq 40$ & $5(6.3)$ & $68(18.9)$ & \\
\hline$>40$ & $74(93.7)$ & $291(81.1)$ & \\
\hline \multicolumn{4}{|c|}{$\begin{array}{l}\text { Tumor size }(\mathrm{cm}), \text { median } \\
(\mathrm{IQR})\end{array}$} \\
\hline Invasive size & $0.60(0.70)$ & $0.70(0.50)$ & 0.338 \\
\hline DCIS size & $0.70(1.40)$ & $1.00(3.00)$ & 0.061 \\
\hline DCIS size $(\mathrm{cm})$ & & & 0.002 \\
\hline$<2$ & $65(83.3)$ & $230(65.0)$ & \\
\hline$\geq 2$ & $13(16.7)$ & $124(35.0)$ & \\
\hline Surgery & & & $<0.0001$ \\
\hline Mastectomy & $26(32.9)$ & $198(55.2)$ & \\
\hline BCS & $53(67.1)$ & $161(44.8)$ & \\
\hline NBR grade & & & 0.247 \\
\hline Gr. 1 & $42(53.2)$ & $159(46.0)$ & \\
\hline Gr. 2,3 & $37(46.8)$ & $187(54.2)$ & \\
\hline ER & & & 0.059 \\
\hline Negative & $17(21.5)$ & $116(32.3)$ & \\
\hline Positive & $62(78.5)$ & $242(67.7)$ & \\
\hline PR & & & 0.342 \\
\hline Negative & 25 (31.6) & $134(37.3)$ & \\
\hline Positive & $54(68.4)$ & $224(62.7)$ & \\
\hline HER2 & & & 0.165 \\
\hline Negative & $62(78.5)$ & $254(70.8)$ & \\
\hline Positive & $17(21.5)$ & $105(29.2)$ & \\
\hline Subtypes & & & 0.257 \\
\hline Luminal A & $26(32.9)$ & $101(28.8)$ & \\
\hline Luminal B & $39(49.4)$ & $149(42.5)$ & \\
\hline Her2 & $9(11.4)$ & $63(17.9)$ & \\
\hline Triple negative & $5(6.3)$ & $38(10.8)$ & \\
\hline \multicolumn{4}{|c|}{$\mathrm{IQR}=$ Interquartile range } \\
\hline \multicolumn{4}{|c|}{ DCIS $=$ Ductal carcinoma in situ } \\
\hline \multicolumn{4}{|c|}{$\mathrm{BCS}=$ Breast Conserving Surgery } \\
\hline \multicolumn{4}{|c|}{ NBR $=$ Nottingham Bloom Richardson } \\
\hline \multicolumn{4}{|c|}{$\mathrm{ER}=$ Estrogen receptor } \\
\hline \multicolumn{4}{|c|}{$\mathrm{PR}=$ Progesterone receptor } \\
\hline HER2 $=$ Human $\mathrm{e}$ & th forto & & \\
\hline
\end{tabular}

Table 3. Multivariate logistic regression model predicting symptomatic $\mathrm{Tl}$ a,bNO breast cancer

\begin{tabular}{lllll}
\hline Variable & \multicolumn{5}{l}{ Detection method } \\
\cline { 3 - 5 } & \multicolumn{5}{c}{ Odds Ratio } & $95 \%$ CI of OR & $p$ value \\
\hline Age (years) & $\leq 40$ & 3.605 & $1.386-9.375$ & 0.009 \\
& $>40$ & 1 & & \\
DCIS size $(\mathrm{cm})$ & & & $1.023-3.918$ & 0.043 \\
& $\geq 2$ & 2.002 & & \\
Surgery & $<2$ & 1 & $1.247-3.725$ & 0.006 \\
& & & & \\
\hline
\end{tabular}

$\mathrm{BCS}=$ Breast Conserving Surgery

DCIS $=$ Ductal carcinoma in situ

$\mathrm{CI}=$ Confidence interval

$\mathrm{OR}=$ Odds ratio
Thirty-eight recurrences were observed after a median follow-up period of 72.9 months, 19 of them distant, and 10 deaths were observed. This corresponded to an overall estimated DRFS rate of $96.9 \%$ at 5 years and $92.4 \%$ at 10 years. The estimated 5 -year and 10-year BCSS rates were $98.4 \%$ and $97.5 \%$, respectively. Univariate analysis showed that symptomatic tumors, large accompanying DCIS lesions, and HER2 positivity were significantly associated with worse DRFS. Tumors accompanied by large DCIS and those that were ER-negative, PR-negative, and HER2-positive resulted in significantly worse BCSS (Table 4).

None of the screening-detected patients experienced distant relapse or death; however, the 10-year DRFS rate for symptomatic cases was $91.1 \%$ $(p=0.049)$. The estimated 10-year BCSS rate was $97 \%$ for symptomatic patients and $100 \%$ for screening-detected patients $(p=0.146)$. Due to lack of events among the screening-detected subgroup of patients (100\% DRFS and 0\% death), the detection method was not included in the multivariate analysis. A large accompanying DCIS lesion size was the only risk factor for poorer DRFS (hazard ratio [HR]: 4.48, $p=0.007$ ) and BCSS (HR: 7.49, $p=0.015)$ in T1a,bN0 breast cancer on multivariate analysis (Table 5). The estimated 10-year DRFS was $97.4 \%$ in patients with small DCIS lesions, but only $77.1 \%$ in patients with large lesions $(p<0.001)$ (Figure 1). The corresponding 10 -year BCSS rates were $97.9 \%$ vs. $94.3 \%$, respectively $(p<0.001)$ (Figure 2).

\section{Discussion}

In this study, we compared the characteristics and survival rates between symptomatic- and screening-detected $\mathrm{T} 1 \mathrm{a}, \mathrm{bN} 0$ breast cancer patients, and found that patients who presented with symptoms were more likely to have large DCIS; this was the only risk factor for distant relapse and worse BCSS.

Approximately $75 \%$ of $\mathrm{T} 1 \mathrm{a}, \mathrm{bN} 0$ breast cancers are currently detected by mammography screening; however, before the prevalence of screening, most $\mathrm{pT} 1 \mathrm{a}, \mathrm{b}$ pN0 breast cancers were discovered owing to symptoms such as a palpable lump. In a study by Lee et al., $64 \%$ of $\mathrm{T} 1 \mathrm{a}, \mathrm{bN} 0$ breast cancer patients diagnosed before 1984 (i.e., before most breast cancer screening programs began) presented with a palpable mass; after 1984, $78 \%$ of the cancers were detected during mammography screenings [7]. Similarly, DCIS was a rare disease discovered in only $2-5 \%$ of all breast cancers before mammography became widespread, usually following clinical symptoms such as a palpable mass, bloody nipple discharge, or Paget's disease [16]. In the current study, large 
accompanying DCIS lesions were found more often in T1aN0 than in T1bN0 breast cancers, likely because the majority $(82 \%)$ of our patients' cancers were symptom-detected. Tumors smaller than $0.5 \mathrm{~cm}$ would not exhibit symptoms unless accompanied by large DCIS lesions.

Table 4. Univariate analysis of survival in patients with $\mathrm{Tla}$,bNO breast cancer

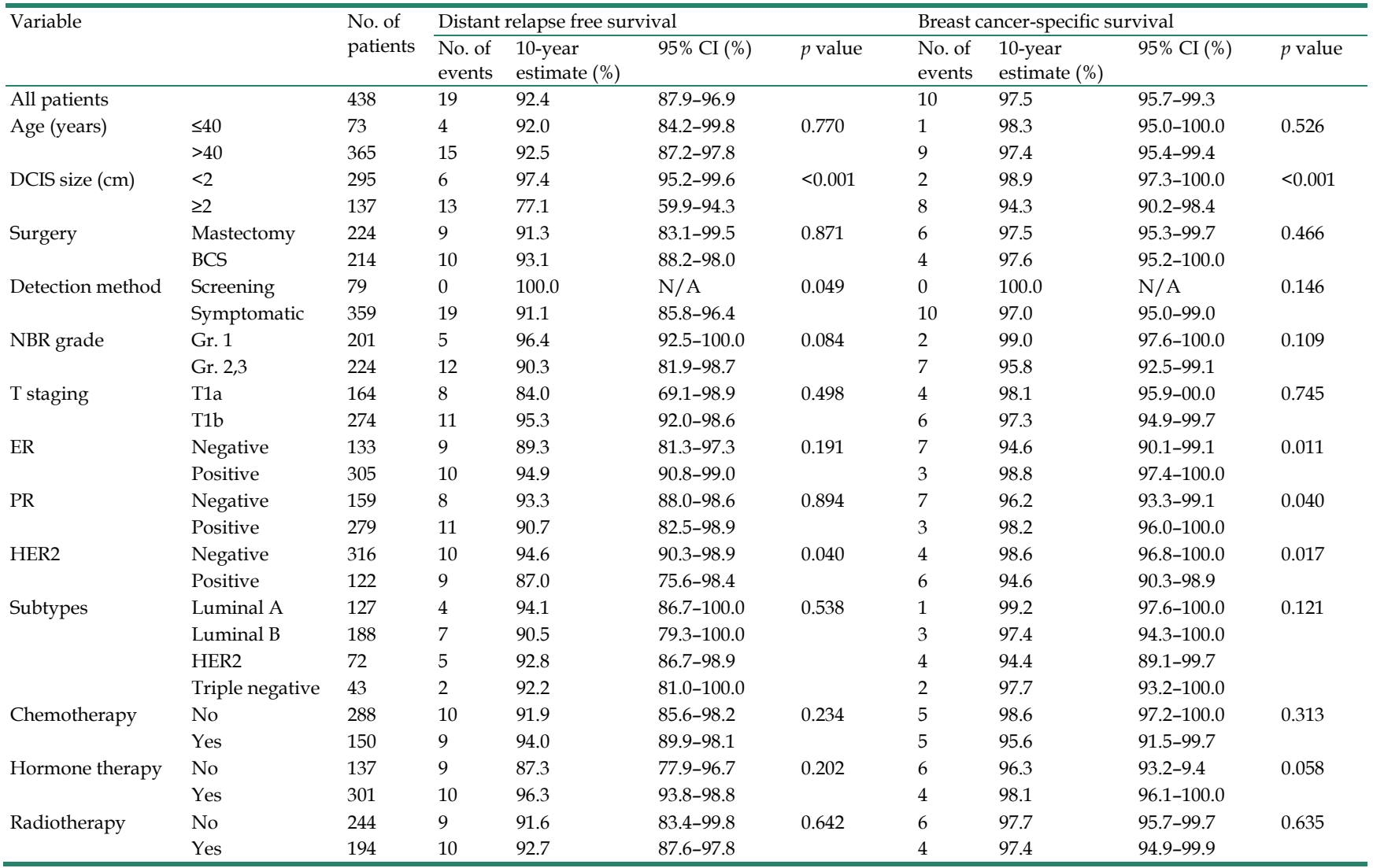

DCIS $=$ Ductal carcinoma in situ

$\mathrm{BCS}=$ Breast conserving surgery

NBR $=$ Nottingham Bloom Richardson

$\mathrm{ER}=$ Estrogen receptor

$\mathrm{PR}=$ Progesterone receptor

HER2 $=$ Human epidermal growth factor receptor 2

$\mathrm{CI}=$ Confidence interval

Table 5. Risk factors for DRFS and BCSS in patients with Tla,bNO breast cancer using multivariate Cox proportional hazards regression analysis

\begin{tabular}{|c|c|c|c|c|c|c|c|}
\hline \multirow[t]{2}{*}{ Variable } & & \multicolumn{3}{|c|}{ Distant relapse free Survival } & \multicolumn{3}{|c|}{ Breast cancer-specific survival } \\
\hline & & Hazard ratio & $95 \% \mathrm{CI}$ of $\mathrm{HR}$ & $P$ value & Hazard ratio & $95 \% \mathrm{CI}$ of $\mathrm{HR}$ & $P$ value \\
\hline DCIS size & $\geq 2 /<2$ & 4.480 & $1.505-13.333$ & 0.007 & 7.491 & $1.479-37.953$ & 0.015 \\
\hline Detection method * & Symptomatic/screening & - & & & - & & \\
\hline NBR grade & Gr. 2,3/Gr. 1 & 1.526 & $0.512-4.546$ & 0.448 & - & & \\
\hline ER & Negative/positive & - & & & 3.092 & $0.370-25.858$ & 0.297 \\
\hline PR & Negative/positive & - & & & 1.393 & $0.186-10.418$ & 0.747 \\
\hline HER2 & Positive/negative & 2.023 & $0.733-5.583$ & 0.174 & 1.490 & $0.326-6.814$ & 0.607 \\
\hline Hormone therapy & No/yes & - & & & 0.639 & $0.094-4.324$ & 0.646 \\
\hline
\end{tabular}

* Excluded variable

DRFS $=$ Distant relapse-free survival

BCSS $=$ Breast cancer-specific survival

DCIS $=$ Ductal carcinoma in situ

NBR $=$ Nottingham Bloom Richardson

$\mathrm{ER}=$ Estrogen receptor

$\mathrm{PR}=$ Progesterone receptor

HER2 = Human epidermal growth factor receptor 2

$\mathrm{CI}=$ Confidence interval

$\mathrm{HR}=$ Hazard ratio 


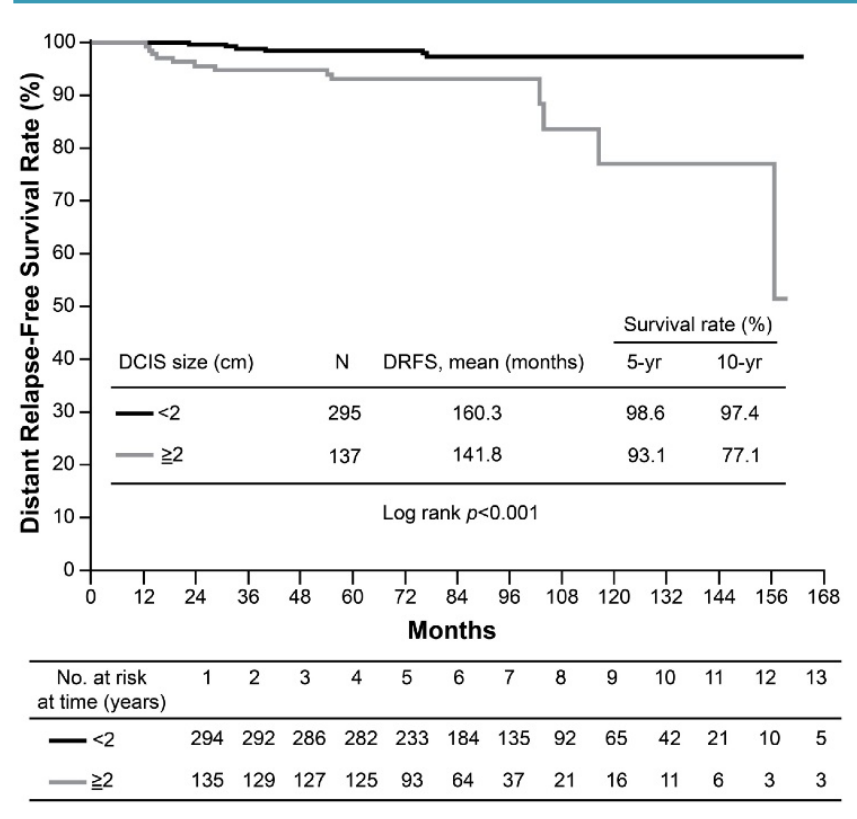

Figure 1. Kaplan-Meier curves for distant relapse-free survival (DRFS) of Tla,bNO breast cancer by ductal carcinoma in situ (DCIS) size.

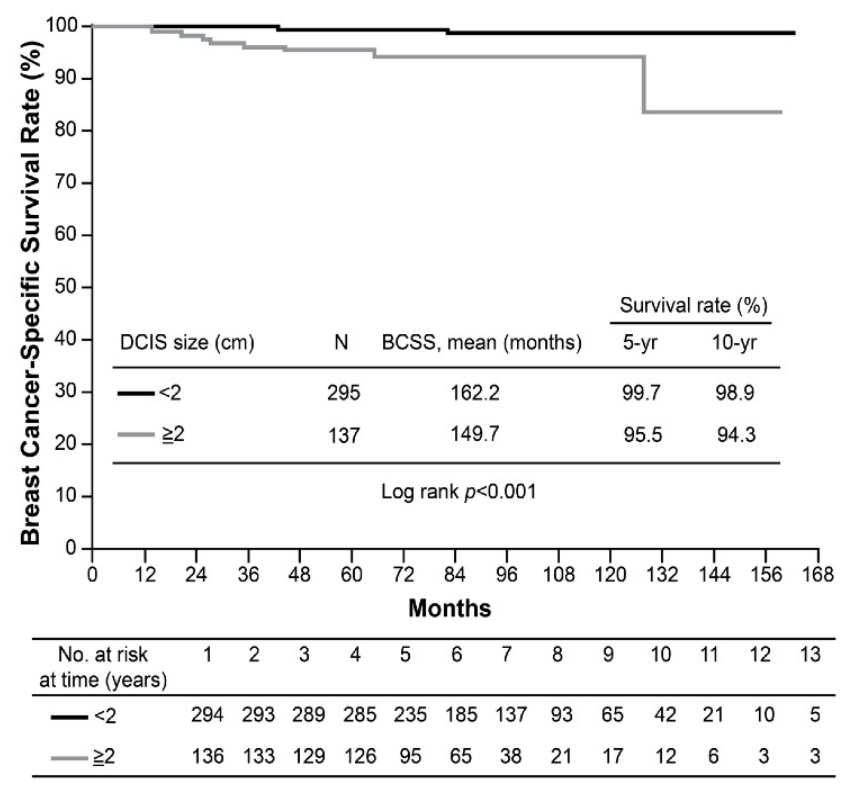

Figure 2. Kaplan-Meier curves for breast cancer-specific survival (BCSS) of $\mathrm{Tla}, \mathrm{bN} 0$ breast cancer by ductal carcinoma in situ (DCIS) size.

Screening-detected breast cancers have consistently had better outcomes [4-6]; however, the detection method has yet to be considered an important prognostic factor. Common arguments against including the detection method include stage shift at diagnosis (lead-time bias) and favorable prognostic factors (length bias) [17-20]; however, there are reports of better outcomes observed for screening-detected breast cancer that cannot be fully explained by these factors. For example, when tumors were stratified into different size or nodal status categories to reduce the magnitude of lead-time bias, screening-detected breast cancers persistently showed better 10-year DRFS and overall survival (OS) [5, 6]. Additionally, a Cox multivariate model that included biologic factors revealed that screening-detected breast cancer remained an independent favorable prognostic factor [5]. These findings are consistent with our observation that screening-detected T1a,bN0 breast cancers have better prognoses; this is not likely to be attributable to either the lead-time effect (because most of the tumors were small and node-negative) or the length effect (because tumor grades and all other aggressive biologic features were not significantly different between screening-detected and symptomatic $\mathrm{T} 1 \mathrm{a}, \mathrm{bN} 0$ breast cancers in our analysis). We also found that symptomatic T1a,bN0 breast cancers are more likely to be associated with large DCIS lesions; large DCIS size was an independent prognostic factor in our multivariate analysis. Thus, large DCIS lesions may at least partially explain the poor prognosis of symptomatic $\mathrm{T} 1 \mathrm{a}, \mathrm{bN} 0$ breast cancers.

The prognosis of DCIS is excellent in general; however, factors such as higher tumor grade, African American ethnicity, comedo necrosis, aggressive biologic subtypes, or presentation with symptoms have all been found to be associated with poorer survival [21-23]. Younger age at diagnosis is also an adverse prognostic factor for DCIS outcomes. It is likely that younger patients rarely undergo screening, and that their cancers are usually detected through symptoms [24]. The prognostic role of the detection method has also been reported in studies of DCIS. In a study of pure DCIS with no adjuvant therapy, DCIS detected by palpation was a risk factor for invasive recurrence [25]. In another study of DCIS by Lagios et al., all of their cases that developed local recurrence or distant metastasis had large DCIS lesions [15]. More recently, an analysis of $>100,000$ women with DCIS from the Surveillance, Epidemiology, and End Results (SEER) database revealed that larger DCIS lesions were found to be associated with worse BCSS rates [26].

There has been little evidence regarding the prognostic significance of associated DCIS in breast cancer. Fehrenbacher et al. noted that several patients in their study of HER2-positive T1a,bN0 breast cancer had large, high-grade DCIS lesions adjacent to small HER2-positive tumors [10]. More recently, Tot reported that the vast majority of the $\mathrm{T} 1 \mathrm{a}, \mathrm{b}$ HER2-positive breast cancers in his study had a high grade, were diffuse, and had extensive in situ components [11]. However, survival analysis regarding the impact of large associated DCIS lesions was not performed in either of these studies.

It remains unclear why large accompanying DCIS lesions are a negative prognostic factor in 
$\mathrm{T} 1 \mathrm{a}, \mathrm{bN} 0$ breast cancers. A possible explanation is that patients with such DCIS lesions may have a greater stromal response, which is directly linked to the risk of invasive cancer recurrence [25]. Second, accompanying DCIS lesions that are large may be more aggressive than their smaller counterparts, just as invasive cancer lesions tend to be more aggressive when they are palpable [27]. Third, larger accompanying DCIS lesions may contain more occult invasive, multicentric components that account for more distant metastasis and shorter survival [15].

Our study has a few limitations. First, our screening-detected group was small $(18 \%$ of the cohort), partly because the nationwide mammographic screening program in Taiwan did not commence until 2004. Most women in Taiwan were not routinely screened during our study period of 2000-2009; the 2 -year screening rate was only $12 \%$ in 2008 [28].

Second, although the median follow-up time is relatively long in our study (approximately 73 months), neither distant recurrence nor death occurred in the screening-detected subgroup. Quiet et al. cautioned on the importance of long-term follow-up in patients with small tumors, which are likely to have a delayed effect on DRFS and OS [29]. Findings consistent with this notion were observed in a recently published study by Houvenaeghel et al. on outcomes of $\mathrm{T} 1$ breast cancer, where the recurrence rate was only $6 \%$ at 5 years but $16.2 \%$ at 10 years [30]. To better estimate the rates of recurrence and death, future research is warranted to collect longitudinal representative data for longer than 10 years, especially from screening-detected patients.

In conclusion, symptomatic $\mathrm{T} 1 \mathrm{a}, \mathrm{bN} 0$ breast cancers are different from those detected by screening, and are associated with larger accompanying DCIS. T1a,bN0 breast cancers accompanying large DCIS lesions are associated with worse survival rates. Our findings suggest that adjuvant systemic therapy should be considered in $\mathrm{T} 1 \mathrm{a}, \mathrm{bN} 0$ breast cancers that are accompanied by large DCIS lesions. Further prospective trials are also warranted.

\section{Abbreviations}

BCSS: breast cancer-specific survival; DCIS: ductal carcinoma in situ; DRFS: distant relapse-free survival; ER: estrogen receptor; HER2: human epidermal growth factor receptor 2; HR: hazard ratio; IHC: immunohistochemistry; NBR: Nottingham Bloom Richardson; PR: progesterone receptor; SEER: Surveillance, Epidemiology, and End Results (database).

\section{Competing Interests}

This research did not receive any specific grant from funding agencies in the public, commercial, or not-for-profit sectors.

The authors have declared that no competing interests exist.

\section{References}

1. Hanrahan EO, Valero V, Gonzalez-Angulo AM, et al. Prognosis and management of patients with node-negative invasive breast carcinoma that is $1 \mathrm{~cm}$ or smaller in size (stage 1; T1a,bN0M0): a review of the literature. J Clin Oncol. 2006; 24: 2113-2122. doi: 10.1200/JCO.2005.02.8035

2. Iqbal J, Ginsburg O, Rochon PA, et al. Differences in breast cancer stage at diagnosis and cancer-specific survival by race and ethnicity in the United States. JAMA. 2015; 313: 165-173. doi: 10.1001/jama.2014.17322

3. Vaz-Luis I, Ottesen RA, Hughes ME, et al. Outcomes by tumor subtype and treatment pattern in women with small, node-negative breast cancer: a multi-institutional study. J Clin Oncol. 2014; 32: 2142-2150. doi: 10.1200/JCO.2013.53.1608

4. Mook S, Van 't Veer LJ, Rutgers EJ, et al. Independent prognostic value of screen detection in invasive breast cancer. J Natl Cancer Inst. 2011; 103: 585-597. doi: 10.1093/jnci/djr043

5. Joensuu H, Lehtimaki T, Holli K, et al. Risk for distant recurrence of breast cancer detected by mammography screening or other methods. JAMA. 2004; 292: 1064-1073. doi: 10.1001/jama.292.9.1064

6. Tabár L, Duffy SW, Vitak B, et al. The natural history of breast carcinoma: what have we learned from screening? Cancer. 1999; 86: 449-462. doi: 10.1002/(SICI)1097-0142(19990801)86:3<449::AID-CNCR13>3.0.CO;2-Q

7. Lee AK, Loda M, Mackarem $G$, et al. Lymph node negative invasive breast carcinoma 1 centimeter or less in size (T1a,bNOMO): clinicopathologic features and outcome. Cancer. 1997; 79: 761-771. doi: 10.1634/theoncologist.2010-0036

8. Wong H, Lau S, Yau T, et al. Presence of an in situ component is associated with reduced biological aggressiveness of size-matched invasive breast cancer. Br J Cancer. 2010; 102: 1391-1396. doi: 10.1038/sj.bjc.6605655

9. Chagpar AB, McMasters KM, Sahoo S, et al. Does ductal carcinoma in situ accompanying invasive carcinoma affect prognosis? Surgery. 2009; 146: 561-567; discussion 567-568. doi: 10.1016/j.surg.2009.06.039

10. Fehrenbacher L, Capra AM, Quesenberry CP Jr., et al. Distant invasive breast cancer recurrence risk in human epidermal growth factor receptor 2-positive T1a and T1b node-negative localized breast cancer diagnosed from 2000 to 2006: a cohort from an integrated health care delivery system. J Clin Oncol. 2014; 32: 2151-2158. doi: 10.1200/JCO.2013.52.0858

11. Tot T. Early $(<10 \mathrm{~mm})$ HER2-positive invasive breast carcinomas are associated with extensive diffuse high-grade DCIS: Implications for preoperative mapping, extent of surgical intervention, and disease-free survival. Ann Surg Oncol. 2015; 22: 2532-2539. doi: 10.1245/s10434-015-4367-9

12. Ho AY, Gupta G, King TA, et al. Favorable prognosis in patients with T1a/T1bN0 triple-negative breast cancers treated with multimodality therapy. Cancer. 2012; 118: 4944-4952. doi: 10.1002/cncr.27480

13. Dieterich M, Hartwig F, Stubert J, et al. Accompanying DCIS in breast cancer patients with invasive ductal carcinoma is predictive of improved local recurrence-free survival. Breast. 2014; 23: 346-351. doi: 10.1016/j.breast.2014.01.015

14. Nair NS, Pandey N, Vanmali V, et al. Should palpable DCIS be treated as IDC? A retrospective audit. J Clin Oncol. 2011; 29: 146. [Meeting Abstract]

15. Lagios MD, Westdahl PR, Margolin FR, et al. Duct carcinoma in situ. Relationship of extent of noninvasive disease to the frequency of occult invasion, multicentricity, lymph node metastases, and short-term treatment failures. Cancer. 1982; 50: 1309-1314 doi: 10.1002/1097-0142(19821001)50:7<1309::AID-CNCR2820500716>3.0.CO;2-\#

16. Julien JP, Bijker N, Fentiman IS, et al. Radiotherapy in breast-conserving treatment for ductal carcinoma in situ: first results of the EORTC randomised phase III trial 10853. EORTC Breast Cancer Cooperative Group and EORTC Radiotherapy Group. Lancet. 2000; 355: 528-533. doi: 10.1016/S0140-6736(99)06341-2

17. Feinstein AR, Sosin DM, Wells CK. The Will Rogers phenomenon. Stage migration and new diagnostic techniques as a source of misleading statistics for survival in cancer. $N$ Engl J Med. 1985; 312: 1604-1608. doi: 10.1056/NEJM198506203122504

18. Connor RJ, Chu KC, Smart CR. Stage-shift cancer screening model. J Clin Epidemiol. 1989; 42: 1083-1095. doi: 10.1016/0895-4356(89)90050-4

19. Kim J, Lee S, Bae $S$, et al. Comparison between screen-detected and symptomatic breast cancers according to molecular subtypes. Breast Cancer Res Treat. 2012; 131: 527-540. doi: 10.1007/s10549-011-1836-0

20. Redondo M, Funez R, Medina-Cano F, et al. Detection methods predict differences in biology and survival in breast cancer patients. BMC Cancer. 2012; 12: 604. doi: 10.1186/1471-2407-12-604 
21. Virnig BA, Tuttle TM, Shamliyan $\mathrm{T}$, et al. Ductal carcinoma in situ of the breast: a systematic review of incidence, treatment, and outcomes. J Natl Cancer Inst. 2010; 102: 170-178. doi: 10.1093/jnci/djp482

22. Leonard GD, Swain SM. Ductal carcinoma in situ, complexities and challenges. J Natl Cancer Inst. 2004; 96: 906-920. doi: 10.1093/jnci/djh164

23. Li CI, Malone KE, Saltzman BS, et al. Risk of invasive breast carcinoma among women diagnosed with ductal carcinoma in situ and lobular carcinoma in situ, 1988-2001. Cancer. 2006; 106: 2104-2112. doi: 10.1002/cncr.21864

24. Esserman L, Yau C. Rethinking the standard for ductal carcinoma in situ treatment. JAMA Oncol. 2015; 1: 881-883. doi: 10.1001/jamaoncol.2015.2607

25. Kerlikowske K, Molinaro A, Cha I, et al. Characteristics associated with recurrence among women with ductal carcinoma in situ treated by lumpectomy. J Natl Cancer Inst. 2003; 95: 1692-1702. doi: 10.1093/jnci/djg097

26. Narod SA, Iqbal J, Giannakeas V, et al. Breast cancer mortality after a diagnosis of ductal carcinoma in situ. JAMA Oncol. 2015; 1: 888-896. doi: 10.1001/jamaoncol.2015.2510

27. Silverstein MI, Skinner KA, Lomis TJ. Predicting axillary nodal positivity in 2282 patients with breast carcinoma. World J Surg. 2001; 25: 767-772. doi: 10.1007/s00268-001-0003-x

28. Yen SM, Kung PT, Tsai WC. Mammography usage with relevant factors among women with mental disabilities in Taiwan: a nationwide population-based study. Res Dev Disabil. 2015; 37: 182-188. doi: 10.1016/j.ridd.2014.10.052

29. Quiet CA, Ferguson DJ, Weichselbaum RR, et al. Natural history of node-negative breast cancer: a study of 826 patients with long-term follow-up. J Clin Oncol. 1995; 13: 1144-1151. doi: 10.1200/JCO.1995.13.5.1144

30. Houvenaeghel G, Goncalves A, Classe JM, et al. Characteristics and clinical outcome of T1 breast cancer: a multicenter retrospective cohort study. Ann Oncol. 2014; 25: 623-628. doi: 10.1093/annonc/mdt532 